\title{
Valve-sparing aortic root replacement in patients with Marfan syndrome - the Homburg experience
}

\author{
Ulrich Schneider, Tristan Ehrlich, Irem Karliova, Christian Giebels, Hans-Joachim Schäfers \\ Department of Thoracic and Cardiovascular Surgery, Saarland University Medical Center, Homburg/Saar, Germany \\ Correspondence to: Univ.-Prof. Dr. med. Hans-Joachim Schäfers, MD. Professor and Chairman, Department of Thoracic and Cardiovascular Surgery, \\ Saarland University Medical Center, Homburg/Saar, Germany. Email: h-j.schaefers@uks.eu.
}

Submitted Jul 03, 2017. Accepted for publication Sep 04, 2017.

doi: 10.21037/acs.2017.11.01

View this article at: http://dx.doi.org/10.21037/acs.2017.11.01

\section{Introduction}

Aortic root dilatation occurs in most patients with Marfan syndrome with secondary valve failure or aortic complications being frequent consequences (1). The traditional surgical approach has been composite replacement of aortic valve and root, first described in the 1960s (2). Since the advent of valve-preserving aortic surgery, different techniques $(3,4)$ have also been applied to Marfan patients. Generally they have resulted in good stability and a low incidence of complications (5-8). In some series, valve reimplantation has resulted in better valve stability at 15 years compared to root remodeling $(5,7)$. This might be due to the fact that this technique-different from remodeling - addresses annular dilatation.

In our own clinical experience, both forms of valve preserving root replacement have led to comparable results (8). As root remodeling has been shown to result in more physiological hemodynamics (9), we have become more liberal to use this approach with time. Beginning in 2004, intraoperative measurement of effective height (10) led to better recognition and more objective treatment of cusp prolapse. Starting 2009 a suture annuloplasty (11) was added to root remodeling in the presence of basal dilatation.

In the present study, we examined the results now reaching 22 years of follow-up. The investigation was based on data collected prospectively and analyzed retrospectively, primary endpoints were freedom from reoperation and from $\mathrm{AR} \geq \mathrm{II}$.

\section{Patients and methods}

Between November 1996 and July 2016, a total of 61 Marfan patients underwent either aortic valve reimplantation $(\mathrm{n}=12$, $19.7 \%)$ or root remodeling $(n=49,80.3 \%)$ at Saarland University Medical Center. All patients were diagnosed with Marfan syndrome according to Ghent criteria (12). The indication for surgery in the majority of patients was ascending aortic aneurysm $(\mathrm{n}=54,88.5 \%)$, in 7 patients $(11.5 \%)$ acute aortic dissection required emergency surgery.

Mean age was $29 \pm 12$ years (range, 2 to 56 years), 36 patients $(59.0 \%)$ were male. The diameter of the basal ring ranged from 18 to $45 \mathrm{~mm}$, the maximum preoperative sinus diameter was $80 \mathrm{~mm}$ (mean $54 \pm 10 \mathrm{~mm}$ ). The sinus was larger in patients undergoing valve reimplantation $(59 \pm 9$ vs. $52 \pm 10 \mathrm{~mm} ; \mathrm{P}=0.03)$. Tricuspid aortic valve anatomy was predominant $(\mathrm{n}=56,91.8 \%), 5$ patients $(8.2 \%)$ had a bicuspid aortic valve. Preoperatively no or just minimal AR was present in 7 patients (11.5\%), it was mild in 17 (27.9\%) and moderate in 16 (26.2\%). In 21 patients (34.4\%) AR was severe (Table 1).

Six patients $(9.8 \%)$ had undergone previous cardiac surgery, additional surgical treatment for cardiac comorbidities was necessary in $16(26.2 \%)$.

All patients were followed clinically and echocardiographically, the degree of AR was determined according to current guidelines (13). The date of first occurrence of recurrent moderate AR was defined as the event when it was first detected.

\section{Surgical technique}

Aortic valve function and root dimensions were examined pre- and post-bypass by intraoperative transesophageal echocardiography. The chest was opened via a median 


\begin{tabular}{|c|c|c|c|}
\hline & Reimplantation & Remodeling & $\mathrm{P}$ \\
\hline$n$ & 12 & 49 & 0.96 \\
\hline Male & $7(58.3 \%)$ & $29(59.2 \%)$ & \\
\hline Female & $5(41.7 \%)$ & $20(40.8 \%)$ & \\
\hline Age (years) & $30 \pm 9$ & $29 \pm 12$ & 0.86 \\
\hline AR pre-OP & & & 0.55 \\
\hline$<1$ & 0 & $7(14.3 \%)$ & \\
\hline I & $3(25 \%)$ & $14(28.6 \%)$ & \\
\hline II & $4(33.3 \%)$ & $12(24.5 \%)$ & \\
\hline III & $5(41.7 \%)$ & $14(28.6 \%)$ & \\
\hline IV & 0 & $2(4.1 \%)$ & \\
\hline Sinus diameter (mm) & $59 \pm 9$ & $52 \pm 10$ & $0.03\left(^{*}\right)$ \\
\hline Basal ring (mm) & $32 \pm 3$ & $31 \pm 4$ & 0.30 \\
\hline \multicolumn{4}{|l|}{ AV-morphology } \\
\hline Tricuspid & $12(100 \%)$ & $44(89.8 \%)$ & 0.25 \\
\hline Bicuspid & 0 & $5(10.2 \%)$ & \\
\hline Central plication & $7(58.3 \%)$ & $42(85.7 \%)$ & $0.03\left(^{*}\right)$ \\
\hline Triangular resection & $1(8.3 \%)$ & 0 & $0.04\left(^{*}\right)$ \\
\hline Pericardial patch insertion & 0 & $2(4.1 \%)$ & 0.48 \\
\hline Suture annuloplasty & 0 & $34(69.4 \%)$ & - \\
\hline Concomitant procedures & & & - \\
\hline Partial arch replacement & $3(25 \%)$ & $5(10.2 \%)$ & \\
\hline Total arch replacement & $1(8.3 \%)$ & $2(4.1 \%)$ & \\
\hline Elephant trunk & $1(8.3 \%)$ & $2(4.1 \%)$ & \\
\hline MVR & $1(8.3 \%)$ & $4(8.2 \%)$ & \\
\hline TVR & 0 & $2(4.1 \%)$ & \\
\hline ASD closure & 0 & $1(2.0 \%)$ & \\
\hline Ablation & 0 & $1(2.0 \%)$ & \\
\hline ECC (min) & $148 \pm 31$ & $105 \pm 29$ & $\left.<0.0011^{(\star \star}\right)$ \\
\hline Myocardial ischemia (min) & $119 \pm 24$ & $78 \pm 19$ & $\left.<0.0011^{(\star \star}\right)$ \\
\hline
\end{tabular}

*, statistically significant; ${ }^{* \star *}$, statistically highly significant. AR, aortic regurgitation; OP, operation; AV, aortic valve; MVR, mitral valve repair; TVR, tricuspid valve repair; ASD, atrial septal defect; ECC, extracorporeal circulation.

sternotomy with right atrial and aortic cannulation. In case of aortic dissection the right axillary artery was used for arterial inflow. The aorta was incised longitudinally followed by transverse opening 5 to $10 \mathrm{~mm}$ above the sinotubular junction, thus carefully maintaining the integrity of aortic valve and commissures. Blood cardioplegia was directly given into the coronary ostia. Stay sutures were placed through the commissures and fixed to 
the patient's chest wall thus preserving the commissural orientation. Since their introduction, valve assessment consisted of measurement of both geometric (14) and effective height (10) to exclude cusp retraction and assess prolapse. The diameter of the basal ring was determined by direct intubation with graded Hegar dilators.

For aortic valve reimplantation, the sinuses of Valsalva were excised and the aortic root mobilized. Subsequently mattress sutures were placed at the level of the basal ring and passed through the proximal end of the vascular graft. The rudimentary aortic root was then fixed inside the graft using a continuous suture carefully placing the commissures at maximum height (15). In root remodeling the sinuses were resected similarly and the Dacron graft was tailored according to root geometry (15). It was subsequently anastomosed to the aortic root following the cusp insertion lines. In both techniques, the coronary buttons were reimplanted into the vascular graft using standard technique.

Initially the diagnosis of cusp prolapse was made by visual inspection. Since 2004 any effective height of $<9 \mathrm{~mm}$ was considered as prolapse and corrected to an effective height of 9 to $10 \mathrm{~mm}$. In addition, care was taken to have all free margins at identical height at the end of the procedure. Cusp correction mainly consisted of central plication $(n=49$, $80.3 \%)$. In case of extreme tissue redundancy a triangular resection was performed and followed by direct adaptation $(\mathrm{n}=1,1.6 \%)$. An autologous pericardial patch that was previously fixed in glutaraldehyde was used for closure of fenestrations $(n=2,3.3 \%)$. Since 2009 a suture annuloplasty to reduce basal dilatation (11) was added to root remodeling if the basal ring exceeded $26 \mathrm{~mm}$ as measured by intubation. It was inserted at the level of the basal ring and subsequently tied around a Hegar dilator according to body surface area ( $21 \mathrm{~mm}: \mathrm{n}=4,23 \mathrm{~mm}: \mathrm{n}=13,25 \mathrm{~mm}: \mathrm{n}=17$ ).

\section{Statistical analysis}

Descriptive statistics are presented as mean \pm standard deviation. Time-dependent data on survival, reoperation and recurrent AR were analyzed using the Kaplan-Meier method. Group comparisons were performed using log-rank test. The significance level was set at $\mathrm{P}<0.05$. Differences between continuous variables were compared by Student's $t$-test or by Welch's $t$-test in case of inhomogeneous variances. Categorical data were compared using chisquare test. All data were analyzed using statistical package SPSS version 20 (SPSS Inc., Chicago, IL, USA), Kaplan-
Meier curves were computed using a commercial available program (Prism, GraphPad Inc., San Diego, CA, USA).

\section{Results}

All aortic valves could be repaired successfully with AR grade I or less at the time of discharge. Mean cardiopulmonary bypass and cross-clamp times were $113 \pm 34$ and $86 \pm 26$ minutes. Both were significantly shorter for root remodeling $(\mathrm{P}<0.001$; Table 1).

No patient died intraoperatively, one death occurred in hospital. In this patient severe depression of left ventricular function was present preoperatively, most likely due to cardiomyopathy. After prolonged cardiac low-output the pediatric patient died from severe heart failure because the parents refused circulatory support or transplantation. Two other patients died long-term, in both patients due to noncardiac reasons. Survival at 5 and 10 years was $98.4 \%$ and 90.8\% after 15 years (Figure 1).

Four patients required reoperation between 26 and 116 months postoperatively. Of those, 3 patients $(75.0 \%)$ had undergone reimplantation previously. Findings at reoperation were recurrent prolapse in 1 patient, cusp retraction in another and commissural detachment after reimplantation for acute aortic dissection in the $3^{\text {rd }}$ patient. Aortic valve repair was feasible in one patient, in the two other valve replacements was performed. The $4^{\text {th }}$ patient was reoperated in another department primarily for severe mitral regurgitation. Due to concomitant recurrent AR grade II the aortic valve was replaced with a mechanical prosthesis. Freedom from reoperation on the aortic valve was $98.2 \%$ at 5 years and $85.0 \%$ at 10 and 15 years. Root remodeling led to increased freedom from reoperation of $95.8 \%$ at 10 years compared to $71.6 \%$ for reimplantation, although the comparison showed only a trend $(\mathrm{P}=0.11$; Figure 2). With suture annuloplasty freedom from reoperation after root remodeling was $100 \%$ at 8 years ( $\mathrm{P}=0.44$; Figure 3$)$.

All patients were discharged with AR grade I or less. At 15 years $40 \%$ of all patients had still no or just trivial AR. Nine patients (14.8\%) developed moderate or more AR over time. Freedom from recurrent $\mathrm{AR} \geq \mathrm{II}$ of $90.7 \%$ at 5 years and $77.7 \%$ at 10 and 15 years. The different techniques led to comparable results. It was $92.8 \%$ at 5 years and $77.3 \%$ at 10 years for remodeling, reimplantation led to $81.8 \%$ at 5 years and $72.7 \%$ at 10 and 15 years $(\mathrm{P}=0.55$; Figure 4$)$. After the addition of suture annuloplasty to root remodeling freedom from recurrent moderate AR was $92.2 \%$ at 5 and 8 years 


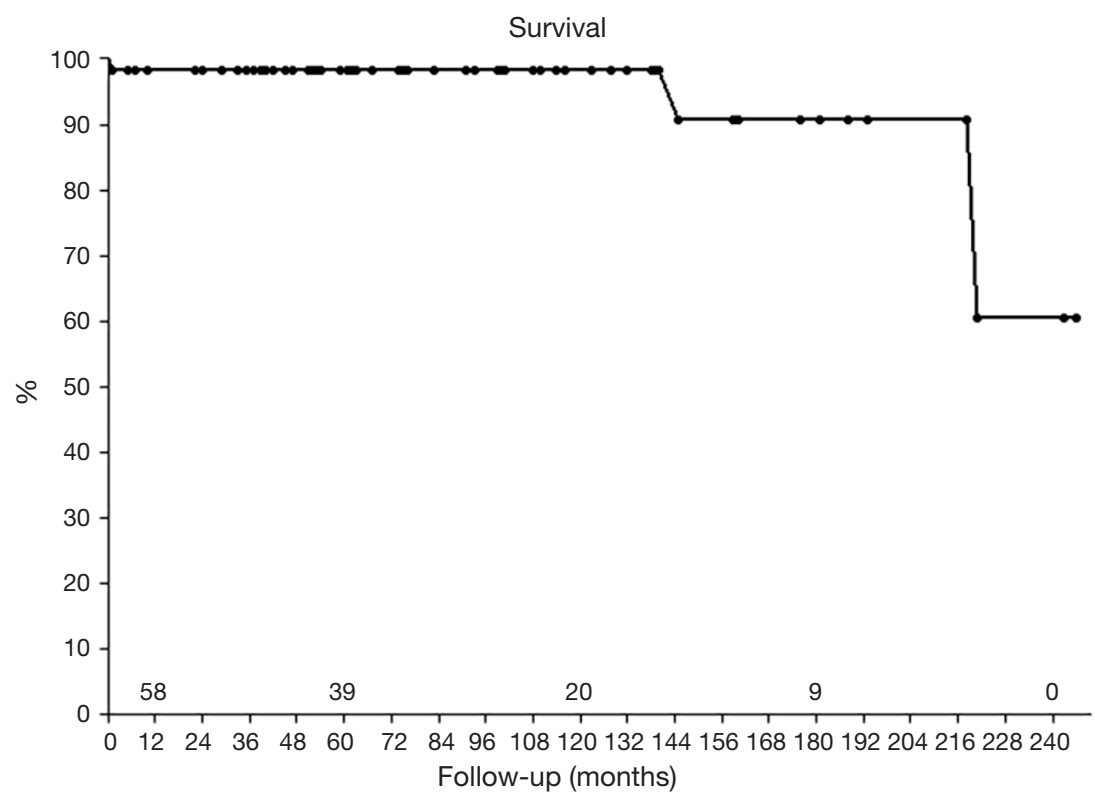

Figure 1 Kaplan-Meier analysis for survival.

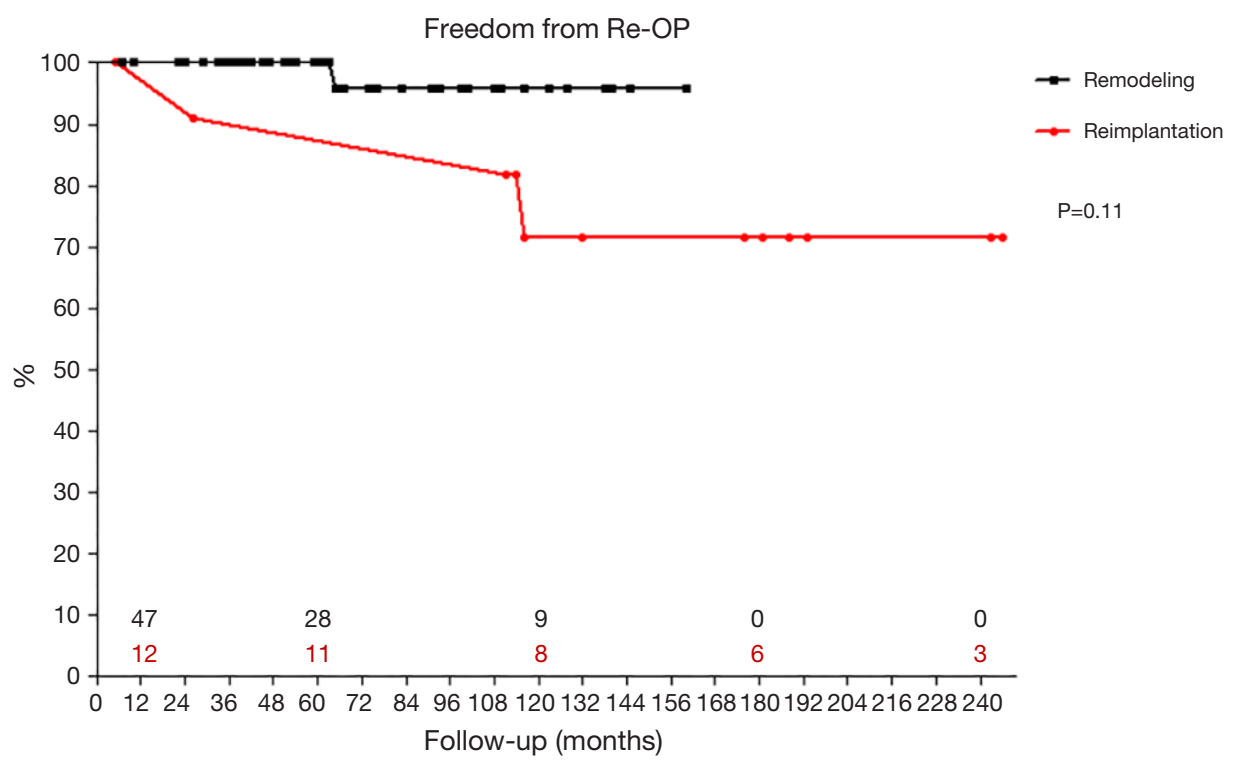

Figure 2 Kaplan-Meier analysis of the 2 groups for freedom from reoperation.

$(\mathrm{P}=0.92)$. Freedom from severe $\mathrm{AR}$ was $91.0 \%$ at 15 years.

\section{Discussion}

Life expectancy of patients with Marfan syndrome is reduced mostly due to the risk of aortic dissection or rupture (1). Composite aortic replacement with a mechanical valve prosthesis (2) historically led to marked improvement of the prognosis. The long-term anticoagulation with its known risks of hemorrhage or thromboembolism is not tolerated by all patients. It may pose particular problems difficult if further aortic interventions are necessary. Finally, composite replacement is associated with a low but existent need for reoperation (16). Bioprostheses or homografts with the known probability of structural deterioration are also not ideal for these generally young patients. 


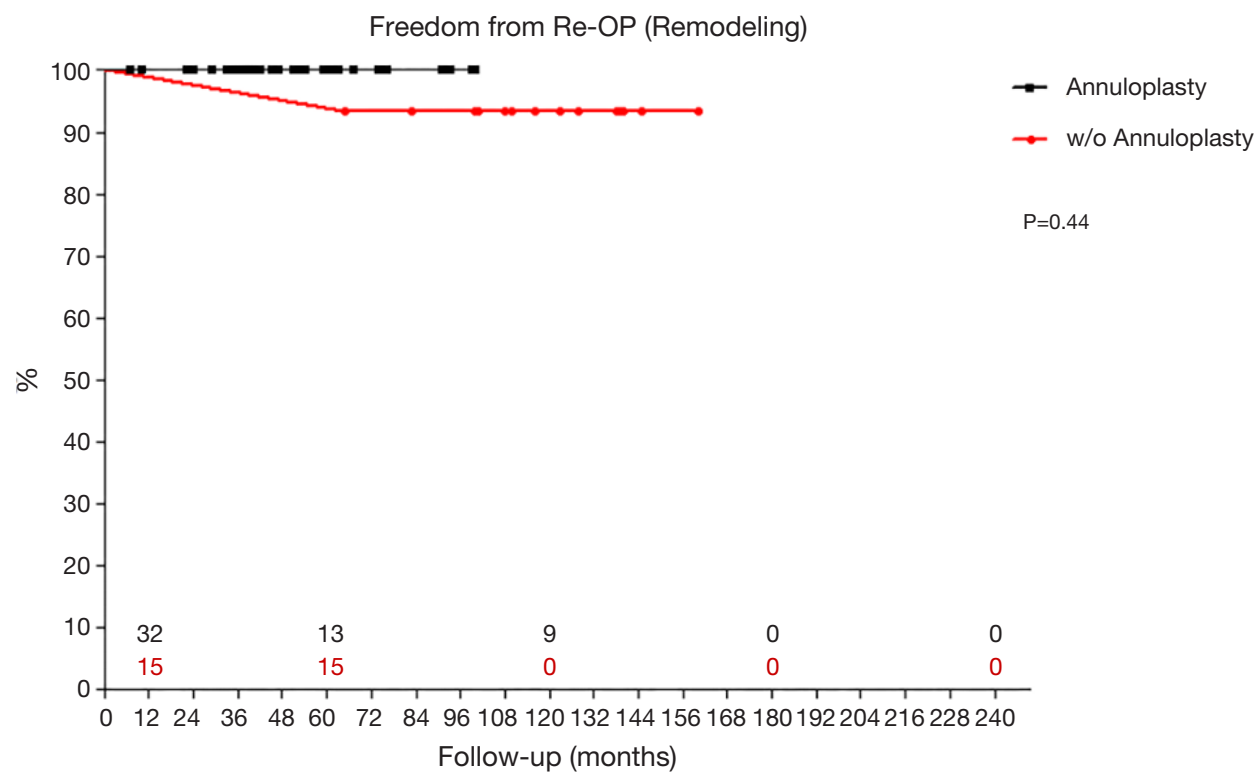

Figure 3 Kaplan-Meier analysis of patients after root remodeling for freedom from reoperation; after addition of an annuloplasty no patient required reoperation.

Freedom from $A R \geq I I$

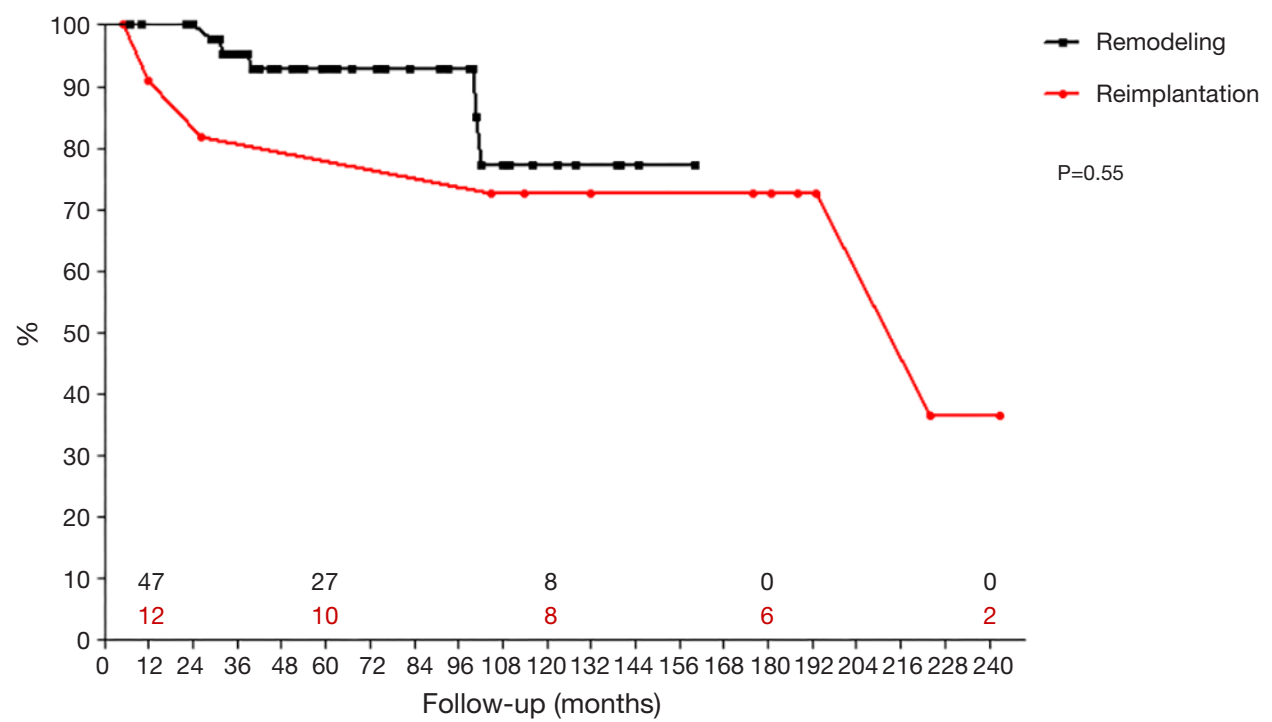

Figure 4 Kaplan-Meier analysis of the 2 groups for freedom from recurrent aortic regurgitation.

Since its introduction, valve-sparing aortic root replacement has become an increasingly accepted alternative in patients with connective tissue disorders. These operations have been shown to result in reduced incidence of thromboembolism and bleeding, albeit at an increased risk of reoperation. Initially two approaches were proposed, valve reimplantation within a vascular graft (3) and root remodeling (4). Between the two, valve reimplantation resulted in apparently better long-term valve stability $(7,17)$; this was attributed to the stabilization of the basal ring (18) by reimplantation, and this technique is recommended in current guidelines (19). In fact, guidelines suggest not to use root remodeling for patients with connective tissue disease, such as Marfan syndrome (19).

A potential disadvantage of valve reimplantation exists in 
the presence of an anatomic variation, i.e., muscle extension into the right coronary sinus or in other terms relevant discrepancy between the level of the basal ring and the aortoventricular junction (18). In this scenario the dissection required for valve reimplantation to anchor the graft at the level of the sinus nadirs will lead to extensive dissection into myocardium with the possibility of entering the right ventricular cavity in a proportion of the cases. If this is to be avoided abortion of valve preservation and instead composite replacement is the logical consequence. It was the occurrence of such scenarios that led us explore the use of root remodeling also in patients with Marfan syndrome. Careful follow up of the patients showed that valve stability was surprisingly good, and this encouraged us to employ the concept further. The more physiologic cusp motion observed after root remodeling (9) reinforced our strategy in our minds, and we hypothesized that reimplantation and remodeling should lead to similar results if normal aortic valve configuration is achieved through the operation.

In an analysis of all patients treated by valve preserving root replacement (i.e., connective tissue disease and those without) we actually observed that the long-term results of reimplantation seemed to be slightly inferior to those obtained with root remodeling (20). Indicators of root size, such as preoperative sinus diameter or basal diameter, seemed to be predictors of suboptimal valve durability (20). At first glance this appeared to confirm the earlier hypothesis (15) that indeed the annular dilatation observed particularly in Marfan patients required more aggressive stabilization. Surprisingly, the results showed no difference between remodeling and reimplantation in patients with annular dilatation. The most likely explanation for the suboptimal valve durability appeared to be the more pronounced size reduction in patients with large annular and root diameter which resulted in more induction of cusp prolapse through more pronounced reduction of intercommissural diameter. In fact, more aggressive correction of cusp prolapse after the introduction of the effective height concept (10) in this subset of patients was associated with significantly better long-term results. Similar observations were made by others (21) who found reduced valve durability in patients with particularly large root size (21). Thus our experience and that of others seems to indicate that cusp prolapse rather than annular dilatation may be the main reason for recurrence of AR. In this context the quantitative assessment of cusp configuration by measurement of effective height rather than simple surgical inspection seems to be an important step towards good short and long-term function of the aortic valve.
The current results support our hypothesis that root remodeling leads to good valve stability also in Marfan patients. Our findings for this technique are comparable to the latest results reported by David and colleagues regarding valve reimplantation (7) or those of Svensson and coworkers (22). Our current approach including precise valve analysis and correction as well as basal stabilization shows almost perfect valve stability during follow-up with the lesser degree of dissection required for root remodeling. We contribute these results to the fact that valve configuration has been measured (10) instead of eye-balled as is done by the majority of groups. Thus generalized prolapse can be avoided. Our findings contradict the current popular assumption described earlier that root remodeling should be abandoned in patients with Marfan syndrome (19). Further long-term follows up will be necessary to confirm the current findings.

\section{Study limitations}

The size of the investigated population limits the relevance of the findings. The strength of this study is that the consistent surgical techniques used minimizes variability due to different surgeons contributing to a clinical series. In addition the number of individuals with a 15 -year follow up is limited even further. A larger study population with long-term follow-up in many patients will make detailed examinations possible. The comparison between root remodeling and valve reimplantation has its drawback in the limited number of patients after valve reimplantation. Thus the described statistical superiority of root remodeling must be considered with caution. Nonetheless, $75 \%$ of patients requiring reoperation had undergone reimplantation previously.

\section{Conclusions}

Valve-sparing aortic root replacement is a valid surgical treatment in patients with Marfan syndrome. Both established forms are feasible and lead to comparable results in long-term follow-up. In our hands, valve reimplantation shows no advantage in terms of reoperation and valve function. Thus we continue to apply root remodeling due to its beneficial effects on valve motion and the lesser degree of surgical trauma. Root remodeling combined with aortic valve repair according to intraoperative effective height and additional basal stabilization by suture annuloplasty in the presence of basal dilatation leads to excellent mid-term results. Longer follow-up will be necessary to confirm these findings after long-term postoperative period. 


\section{Acknowledgements}

None.

\section{Footnote}

Conflicts of Interest: The authors have no conflicts of interest to declare.

\section{References}

1. Marsalese DL, Moodie DS, Vacante M, et al. Marfan's syndrome: natural history and long-term followup of cardiovascular involvement. J Am Coll Cardiol 1989;14:422-8; discussion 429-31.

2. Bentall H, De Bono A. A technique for complete replacement of the ascending aorta. Thorax 1968;23:338-9.

3. David TE, Feindel CM. An aortic valve-sparing operation for patients with aortic incompetence and aneurysm of the ascending aorta. J Thorac Cardiovasc Surg 1992;103:61721; discussion 622.

4. Sarsam MA, Yacoub M. Remodeling of the aortic valve anulus. J Thorac Cardiovasc Surg 1993;105:435-8.

5. Patel ND, Weiss ES, Alejo DE, et al. Aortic root operations for Marfan syndrome: a comparison of the Bentall and valve-sparing procedures. Ann Thorac Surg 2008;85:2003-10; discussion 2010-1.

6. Karck M, Kallenbach K, Hagl C, et al. Aortic root surgery in Marfan syndrome: Comparison of aortic valve-sparing reimplantation versus composite grafting. J Thorac Cardiovasc Surg 2004;127:391-8.

7. David TE, David CM, Manlhiot C, et al. Outcomes of Aortic Valve-Sparing Operations in Marfan Syndrome. J Am Coll Cardiol 2015;66:1445-53.

8. Kunihara T, Aicher D, Rodionycheva S, et al. Outcomes after valve-preserving root surgery for patients with Marfan syndrome. J Heart Valve Dis 2012;21:615-22.

9. Fries R, Graeter T, Aicher D, et al. In vitro comparison of aortic valve movement after valve-preserving aortic replacement. J Thorac Cardiovasc Surg 2006;132:32-7.

10. Schäfers HJ, Bierbach B, Aicher D. A new approach to the assessment of aortic cusp geometry. J Thorac Cardiovasc Surg 2006;132:436-8.

11. Schneider U, Aicher D, Miura Y, et al. Suture Annuloplasty in Aortic Valve Repair. Ann Thorac Surg 2016;101:783-5.

12. Loeys BL, Dietz HC, Braverman AC, et al. The revised Ghent nosology for the Marfan syndrome. J Med Genet 2010;47:476-85.
13. Lancellotti P, Tribouilloy C, Hagendorff A, et al, European Association of Echocardiography. European Association of Echocardiography recommendations for the assessment of valvular regurgitation. Part 1: aortic and pulmonary regurgitation (native valve disease). Eur J Echocardiogr 2010;11:223-44.

14. Schäfers HJ, Schmied W, Marom G, et al. Cusp height in aortic valves. J Thorac Cardiovasc Surg 2013;146:269-74.

15. Schäfers H, Fries R, Langer F, et al. Valve-preserving replacement of the ascending aorta: remodeling versus reimplantation. J Thorac Cardiovasc Surg 1998;116:990-6.

16. Mookhoek A, Korteland NM, Arabkhani B, et al. Bentall Procedure: A Systematic Review and Meta-Analysis. Ann Thorac Surg 2016;101:1684-9.

17. Benedetto U, Melina G, Takkenberg JJ, et al. Surgical management of aortic root disease in Marfan syndrome: a systematic review and meta-analysis. Heart 2011;97:955-8.

18. Anderson RH. Clinical anatomy of the aortic root. Heart 2000;84:670-3.

19. Hiratzka LF, Bakris GL, Beckman JA, et al. 2010 ACCF/ AHA/AATS/ACR/ASA/SCA/SCAI/SIR/STS/SVM guidelines for the diagnosis and management of patients with Thoracic Aortic Disease: a report of the American College of Cardiology Foundation/American Heart Association Task Force on Practice Guidelines, American Association for Thoracic Surgery, American College of Radiology, American Stroke Association, Society of Cardiovascular Anesthesiologists, Society for Cardiovascular Angiography and Interventions, Society of Interventional Radiology, Society of Thoracic Surgeons, and Society for Vascular Medicine. Circulation 2010;121:e266-369.

20. Kunihara T, Aicher D, Rodionycheva S, et al. Preoperative aortic root geometry and postoperative cusp configuration primarily determine long-term outcome after valve-preserving aortic root repair. J Thorac Cardiovasc Surg 2012;143:1389-95.

21. Miyahara S, Matsueda T, Izawa N, et al. Mid-Term Results of Valve-Sparing Aortic Root Replacement in Patients With Expanded Indications. Ann Thorac Surg 2015;100:845-51; discussion 852.

22. Svensson LG, Blackstone EH, Alsalihi M, et al. Midterm results of David reimplantation in patients with connective tissue disorder. Ann Thorac Surg 2013;95:555-62.

Cite this article as: Schneider U, Ehrlich T, Karliova I, Giebels C, Schäfers HJ. Valve-sparing aortic root replacement in patients with Marfan syndrome-the Homburg experience. Ann Cardiothorac Surg 2017;6(6):697-703. doi: 10.21037/ acs.2017.11.01 